\title{
Los principios de la fenomenología y la fenomenología de lo inaparente. Aspectos del método en las filosofias de Michel Henry y Jean-Luc Marion
}

\author{
Hernán Inverso \\ Universidad de Buenos Aires
}

\begin{abstract}
Resumen: Este trabajo estudia los desarrollos de M. Henry y J.-L. Marion a propósito de los principios de la fenomenología, su número y su función. Para ello revisa los argumentos que llevan a replantear su vinculación y sostienen la propuesta de estos autores de elevar su número. Finalmente, analiza la máxima "a las cosas mismas", como quintaesencia fenomenológica, a los efectos de relevar las claves que ofrece para el planteamiento de una fenomenología de lo inaparente y su consecuente ampliación del entorno de fenómenos aludidos.
\end{abstract}

Palabras clave: fenomenología; principios; método; Jean-Luc Marion; Michel Henry

\begin{abstract}
The Principles of Phenomenology and the Phenomenology of the Inapparent. Aspects of the Method in the Philosophies of Michel Henry and Jean-Luc Marion". This article studies the developments made by M. Henry and J.-L. Marion regarding the principles of phenomenology, their number and function. In order to accomplish this, we will review the arguments that lead these authors to rethink the relationship of these principles and sustain the authors' proposal of increasing its number. Finally, we will analyze the maxim "to the things themselves" as a phenomenological quintessence, in order to discover the keys it provides for a phenomenology of the inapparent and its consequent expansion of the phenomena studied by phenomenology.
\end{abstract}

Keywords: phenomenology; principles; method; Jean-Luc Marion; Michel Henry 
Jean-Luc Marion presentó en Reducción y donación (1989) su proyecto de resignificación del programa fenomenológico. A propósito de esta obra, Michel Henry propuso en "Quatre principes de la phénoménologie" (1991) una revisión del dispositivo tradicional teniendo en cuenta la adición del principio marioniano "a tanta reducción, tanta donación" en último lugar. Allí, Henry señala una serie de inconvenientes del planteamiento de Husserl sosteniendo que los principios tradicionales pretenden radicalidad, pero en realidad permanecen indeterminados, lo cual afecta el destino de la fenomenología íntegra hasta el punto de caer en contradicción ${ }^{1}$. La indeterminación constituiría un problema que persigue a Husserl y deriva de la conjunción entre intencionalidad y constitución, dejando al enfoque íntegro presa de la comprensión de la manifestación solo a la manera de un fenómeno mundano ${ }^{2}$.

Frente a esto, sugeriremos que los núcleos de estas críticas henrianas, a las que pueden sumarse las de Marion, aluden a lecturas que pasan por alto -en parte debido a los avatares de la publicación de la Husserliana- puntos importantes del constructo teórico husserliano, condenando la posición general con afirmaciones que tienen validez en el terreno específico de una determinada dimensión fenomenológica, pero que resultan inaplicables a la totalidad del planteamiento. El resultado es una visión sesgada, construida con elementos de los enfoques estático y genético, sin incorporar otros desarrollos asociados con la generatividad. Una vez desplegado el concierto íntegro de modalidades de apertura a los fenómenos que están in nuce en Husserl, es posible incorporar los objetivos de estas filosofias que reclaman radicalidad en el plexo de la fenomenología de lo inaparente.

Para identificar los puntos que permiten esta torsión, bosquejaremos primero la posición de Henry respecto de los principios de la fenomenología y su diagnóstico crítico, deteniéndonos en su lectura del cuarto principio, de factura marioniana. A continuación, analizaremos el contexto en que Marion postula esta idea para examinar finalmente el lema "a las cosas mismas" y sus implicancias para la relación entre fenomenología y excedencia. Este recorrido nos permitirá sopesar los aportes de esta discusión en el marco de la fenomenología de lo inaparente y su relación con la matriz husserliana.

Cf. Henry, M., Phénoménologie de la Vie, 1 (en adelante, PhV), París: PUF, 2003, pp. 78-104.

2 Cf. Henry, M., Phénoménologie materielle, París: PUF, 1990, p. 8. 


\section{Los principios y su número en la interpretación henriana}

El texto "Quatre principes de la phénoménologie" comienza con un listado que oficia como presentación. El primer principio se remonta a la formulación heideggeriana de "a tanta apariencia, tanto ser". Husserl lo enunció de una manera amplia: "soviel Schein, soviel... Sein” para marcar la correlación entre el ser del mundo y el modo como se da a la conciencia, indicando que toda afirmación sobre el mundo depende de los modos de donación. Henry propone expresarlo en infinitivo - "a tanto aparecer, tanto ser"- para evitar la equivocidad de la noción de apariencia, que mienta, a la vez, el contenido que aparece y el acto de aparecer ${ }^{4}$. Como indica L. Stan, la adopción de este inicio, pero a la vez la idea de su limitación (en tanto diluye la importancia del estudio del puro hecho del aparecer), caracteriza bien la posición de Henry ${ }^{5}$.

El segundo principio, que Husserl llama en Ideas I, §24, “principio de todos los principios", plantea que "toda intuición donadora es una fuente de derecho para el conocimiento, de modo que todo lo que se ofrece originariamente a nosotros en la intuición... debe ser simplemente tomado por lo que se da"6. En el contexto husserliano, este principio se asocia a la intuición de esencias, pero se puede vincular, de la misma manera, con los planteamientos de La idea de la fenomenología que conectan evidencia y donación ${ }^{7}$.

El tercero es el lema zu den Sachen selbst, que Henry vierte como "droit aux choses mêmes!" al distinguir la filosofia de las ciencias empíricas y las matemáticas, señala la importancia de apelar a las cosas mismas ${ }^{9}$.

El cuarto principio, finalmente, es introducido por Marion en Reducción y donación: "a tanta reducción, tanta donación". Nos detendremos en él más adelante. Por ahora, basta señalar que este principio enfatiza la dimensión del

\footnotetext{
3 Hua I, p. 133. La sigla Hua, con indicación de tomo y página, corresponde a Husserl, Edmund, Gesammelte Werke-Husserliana, 42 vv., Biemel, W. y otros (eds.), Dordrecht: Springer (con anterioridad, Kluwer Academic Publishers y Martinus Nijhoff), 1950-2014.

4 Cf. Popa, D., "Michel Henry, lecteur de Husserl. Apparence, phénoménalité et présence à soi", en: Cahiers philosophiques, v. III, 126 (2011), pp. 82-94.

5 Cf. Stan, L., "Michel Henry: The Goodness of Living Affectivity", en: Stewart, J. B. (ed.), Kierkegaard and Exitentialism, Surrey: Ashgate, 2011, p. 130.

6 "jede originar gebende Anschauung eine Rechtquelle der Erkenntnis sei, daß alles, was sich uns inter Intuition originär... darbietet, einfach hinzunehmen sei, als was es sich gibt" (Hua III/1, p. 51).

Hua II, p. 73. Cf. Inverso, H., Fenomenologia de lo inaparente, Buenos Aires: Prometeo, 2018, pp. 31-48.

8 Henry, M., Phénoménologie de la Vie, p. 77.

9 Hua XIX/1, p. 10.
} 
aparecer de un modo que escapa a los compromisos ontológicos de la primera formulación, a la vez que resalta la importancia del dispositivo metodológico reductivo.

La revisión henriana tiene como propósito mostrar que este dispositivo presenta inconvenientes que lo hacen inviable. El primer principio establece una correlación entre aparecer y ser que se resuelve en identidad de esencia y requiere, por tanto, ser cuestionado, dado que la dignidad ontológica de ambos elementos es divergente. Al contrario, el ser se funda en el aparecer, base sobre la cual, desde los planteamientos originarios, se apoya la pretensión de prioridad de la fenomenología sobre la ontología. Husserl trazaria, en cambio, una asociación donde quedan en fusión indiferenciada y, en ese sentido, se produce una contaminación ontológica que socava la formulación y que finalmente lleva a su ocaso.

Asimismo, en el tercer principio, Henry interpreta una dualidad entre la preposición $z u$, con idea de direccionalidad, y el núcleo die Sachen, que separaría el acceso y el contenido al que se da acceso. En la remisión a las cosas hay un riesgo de nueva caída en la indistinción entre aparecer y ser: el ser no es reductible al aparecer porque el aparecer no confiere el ser. Por el contrario, en la direccionalidad hacia la cosa adviene un dominio del objeto que "decide el medio de ser conocido" e impone una dependencia de la fenomenología respecto de la ontologia ${ }^{10}$. La salida es taxativa: "a la afirmación según la cual es la naturaleza de la cosa la que decide los medios de acceder a ella, no conviene responder"11. Al contrario, hay que advertir que la cosa se descubre en su aparecer y en el practicar la reducción del ser al aparecer, que resulta primario y libera la noción de ser de cualquier contaminación óntica.

Se trata de la misma disociación peligrosa que se detecta en la noción de fenómeno, si se concibe que el ser es independiente antes de mostrarse. El rumbo señalado frente a esto será la reducción del ser al aparecer del aparecer, que conduce a la tematización de la confusión entre el aparecer del mundo y la esencia de la manifestación ${ }^{12}$. Esta crítica alcanza al tercer principio, que no es radical porque, en rigor, no explica el aparecer y supone que la intencionalidad se dirige a un afuera trascendente en una puesta a distancia que se extiende

${ }^{10}$ Cf. Henry, M., Phénoménologie de la Vie, p. 81.

${ }^{11}$ Ibid.

${ }^{12}$ Cf. García Jarama, J., Finitud, carne e intersubjetividad, Toledo: San Idelfonso, 2007, p. 113. 
a todo fenómeno. De este modo, resulta incumplimentable porque remite a un plexo de cosas que requiere el aparecer mismo aún no elucidado13.

Esta crítica se conecta con la que Henry vierte en "La inversión de la fenomenología", donde cuestiona la lógica de la visión que prevalece en el modelo fenomenológico y caracteriza el hacer ver como una función de puesta a distancia ${ }^{14}$. Esto quiere decir que el compromiso con la intencionalidad reposa en el hacer ver que revela un objeto, entendido etimológicamente como "lo puesto adelante" y, por tanto, el modo de aparecer del objeto queda asociado con la puesta a distancia (mise à distance). En efecto, al depender de la intencionalidad, señala Henry, la fenomenalidad resulta constituida por el hacer ver y de ahí surge la aporía de que la intencionalidad no puede referirse a sí misma. Así, queda afectada por un defecto que la anula como mecanismo para dar cuenta de los elementos de la subjetividad de la vida como sujeto último omniabarcante ${ }^{15}$.

Con la fórmula de que la visión "a jamais vu sa propre vision", Henry impugna dos puntos importantes de la fenomenología husserliana: por un lado, la reflectio, que queda afectada por sus efectos incompletos y ciegos a su propio mecanismo; por otro, la apelación a la intuición que, como dispositivo de la intencionalidad, queda en entredicho y, con ello, condiciona la legitimidad del segundo principio: el principio de todos los principios ${ }^{16}$. En suma, la intencionalidad reduciría todo aparecer a la estructura noético-noemática e imposibilitaría toda elucidación a través del aparecer, que debería ser el objeto prioritario en una investigación fenomenológica. Sin embargo, del mismo modo, la intencionalidad es tal solo sobre la base de la no intencionalidad de la Vida, que es fundamento y condición de todo lo demás. La esencia de la vida, según Henry, radicará en esta auto-afección patética no afectada por nada diferente de sí y de manera radical, no mediante un sentido interno o externo y, a la vez, de manera inmanente en tanto excluye toda intencionalidad y toda trascendencia $^{17}$. Esta caracterización de la vida invisible se torna posible gracias a la afectividad, que le permite a la vida -y a los vivientes- sentirse y experi-

${ }^{13}$ Cf. Henry, M., Phénoménologie de la Vie, p. 84.

${ }^{14}$ Cf. Henry, M., Incarnation. Une philosophie de la chair, París: Éditions du Seuil, 2000, p. 51.

${ }^{15}$ Cf. Henry, M., Incarnation. Une philosophie de la chair, I, p. 54. Sobre este aspecto en relación con la ekstaticidad, $c f$. Canullo, C., "Ekstasy of the World/Immanence of Life. Michel Henry reader of Husserl”, en: Analecta Husserliana, Nueva York: Springer, 2003.

${ }^{16}$ Cf. Henry, M., Incarnation. Une philosophie de la chair, p. 52. Cf. García Jarama, J., Finitud, carne e intersubjetividad, p. 113.

17 Sobre la noción de auto-afección como base de la noción del enfoque henriano, cf. más adelante, el Capítulo 3 y el trabajo de N. Depraz y B. Michel. 
mentarse a sí misma, sin la intermediación de un sentido o el establecimiento de una distancia.

Esta idea ha recibido varias criticas. Entre ellas, A. Calcagno rechaza el planteamiento henriano diciendo que nada impide tornar la vida en un objeto intencional sujeto a variación eidética para arribar a su esencia y que, de hacerlo, varios de sus aspectos resultarian dudosos respecto de su carácter de fundamento. En esta línea, enfatiza que la perspectiva husserliana está efectivamente abierta a la donación, pero su perspectiva no es exhaustiva porque se atendría a los límites del conocimiento humano ${ }^{18}$. En apoyo de la misma idea, P. MacDonald apela al planteamiento levinasiano sobre la conciencia no agotada por la intencionalidad como enseñanza esencial de Husserl ${ }^{19}$.

En efecto, es objetable la idea de que la fenomenología husserliana no puede tematizar la intencionalidad, cosa que de hecho hace al remontarse a sus planos fundantes en la dimensión de la génesis, la generatividad y la inapariencia. Los tres primeros, comprendidos a la manera de estratos fenomenológicos, han sido tematizados por el mismo Husserl o puestos de relieve por la crítica posterior ${ }^{20}$. En la misma línea, es posible reunir como estrato de lo inaparente los desarrollos husserlianos y post-husserlianos que toman como fenómeno de su atención prioritaria la donación y la excedencia ${ }^{21}$. Desde esta perspectiva, cada enfoque fenomenológico da cuenta de los fenómenos desde su propia legalidad, por lo cual un mismo fenómeno puede resultar llamativamente distinto si se lo analiza desde uno u otro. Se puede hacer, por ejemplo, la experiencia del acontecimiento y pensar la entrada de Napoleón a Jena

\footnotetext{
${ }^{18}$ Cf. Calcagno, A., "Michel Henry's Non Intentionality Thesis and Husserlian Phenomenology", en: Journal of the British Society for Phenomenology, v. XXXIX, 2 (2008), p. 118.

${ }^{19}$ Cf. MacDonald, P., Languages of Intentionality: A Dialogue Between Two Traditions on Consciousness, Londres: Continuum, 2012, p. 194. En Más allá de la intencionalidad, Levinas señala los aspectos pasivos y activos de la intencionalidad derivados de la encarnación en un cuerpo, donde intencionalidad significa una exterioridad en la inmanencia y la inmanencia de toda exterioridad ( $c f$. Levinas, E., "Beyond Intentionality", en: Montefiore, A. (ed.), Philosophy in France Today, Cambridge: Cambridge University Press, 1983, p. 106).

${ }^{20}$ Sobre los enfoques estático y genético y el planteamiento de la dimensión generativa, $c f$. Steinbock, A., Home and Beyond: Generative Phenomenology After Husserl, Illinois: Northwestern University Press, 1995; Steinbock, A., "Husserl's Static and Genetic Phenomenology: Translator's Introduction to two Essays", en: Continental Philosophy Review, 31 (1998) y Steinbock, A., "Generativity and the Scope of Generative Phenomenology", en: Welton, D., The New Husserl: A Critical Reader, Indiana: Indiana University Press, 2003.

${ }^{21}$ Cf. Walton, R., "Teleologia y teologia en Edmund Husserl", en: Estudios de filosofia, 45 (2012), que llama a esta dimensión "metahistoria", e Inverso, H., "La fenomenología de lo inaparente y el problema de las vías hacia el plano trascendental", en: Eidos, 26 (2016) y Fenomenología de lo inaparente, Buenos Aires: Losada, 2018, a propósito de la fundamentación para el planteamiento de un estrato de lo inaparente, punto en el que aquí no nos detenemos.
} 
como fenómeno initerable e inagotable en su excedencia, pero para continuar su análisis en la historicidad la investigación se plasma en sede generativa, o incluso genética o estática si la tematización lleva a estos ámbitos.

El resultado de estos análisis no es en sí mismo definitivo dado que, si bien todos se dirigen a las cosas mismas y toman de ellas los rasgos que efectivamente se dan, las modalidades intencionales enfocan ciertos rasgos y dejan otros en situación de opacidad. De esta manera, no es un problema del método ni una confusión funesta que la vida u otros fenómenos asociados con la donación última y el plano del fundamento aparezcan bajo perspectivas sesgadas, dado que ese sesgo es producto del peculiar recorte intencional del tipo de investigación en cuestión. Es por ello que la función del acto intencional del polo subjetivo se silencia metodológicamente en el plano de la fenomenología de lo inaparente, a diferencia de los otros donde conserva su actividad, precisamente porque en este punto el objetivo prioritario es dejar aparecer al aparecer mismo.

Las discusiones y confusiones a propósito de la subjetividad surgen, en buena medida, de omitir la peculiaridad de las condiciones metodológicas de la fenomenología de lo inaparente, que no condenan al sujeto a la pasividad, sino que crean el marco necesario (la puesta en anamorfosis, para usar categorias marionianas) para abrirse a la donación originaria ${ }^{22}$. En este sentido es un mecanismo que enfatiza el dominio del objeto, pero que no contraviene la actitud fenomenológica por definición activa.

Esta omisión es la que lleva a Marion a proponer un "contra-método". Si la fenomenología queda redefinida de tal modo que su objetivo prioritario es acceder al aparecer del aparecer, resulta necesario asegurar que este se muestre, es decir, evitar todo bloqueo de la mostración. No obstante, Marion sugiere que un método implicaría el control subjetivo y, por tanto, resultaría contraproducente y entorpecería el camino del fenómeno. Se necesitaría, entonces, una reducción que abra "el espectáculo del fenómeno" y se retraiga como un "giro que gira contra sí mismo y consiste en esa vuelta misma" 23 .

Sin embargo, esto no se aplica a la fenomenología en general, sino a su estrato orientado a la inapariencia. El predominio del objeto constituye un

\footnotetext{
22 Sobre la anamorfosis, cf. Marion, J.-L., Étant donné. Essai d'une phénoménologie de la donation, París: PUF, 1997, p. 217, e Inverso, H., "Polo subjetivo y toma de iniciativa. La fenomenología de lo inaparente y el contra-método marioniano", en: Revista Escritos, 56 (2018), pp. 63-82.

23 "tournant, qui tourne contre elle-même et consiste en ce retournement lui-même" (Marion, J.-L., Étant donné. Essai d'une phénoménologie de la donation, p. 17).
} 
dispositivo dentro del método, que apela a recursos y vías muy distintas de acuerdo con el tipo de apertura a la donación que se pone en juego. En efecto, la excedencia adviene en múltiples sectores de la investigación fenomenológica: lo estático y su relación con la inagotabilidad de perspectivas, lo genético en campos como la conciencia interna del tiempo y la sintesis pasiva, y la generatividad y su relación con lo inexhaustible de los fenómenos históricos. Tematizarla es aplicar los dispositivos ya existentes para dar cuenta de algo que siempre estuvo ahí, como una llamada a la espera de una respuesta que encuentra en el estrato de inapariencia su lugar propio.

Esta misma idea subyace en la declaración de Husserl a Dietrich Mahnke en una carta de 1917, donde reconoce los distintos estratos y la atención brindada a unos sin desmerecer el peso de aquellos que recibieron menos tratamiento y conciernen precisamente a la excedencia ${ }^{24}$. En este sentido, la configuración de un espectro fenomenológico de cuatro estratos, con la dimensión de lo inaparente recuperada como plano legítimo, responde a proyecciones eminentemente husserlianas. La confirmación viene de la mano de las Meditaciones cartesianas, que nos muestran a Husserl señalando hacia este camino, cuando afirma: "con esto, por cierto, no se rozan aún los problemas generativos... los cuales manifiestamente pertenecen a una dimensión superior y presuponen un trabajo de explicitación tan extraordinario de las esferas inferiores, que ellos, todavía por mucho tiempo, no podrán plantearse como problemas sobre los que se pueda trabajar" 25 .

Las dimensiones superiores necesitan, entonces, un trabajo previo exhaustivo y es solo sobre esta base que cobran sentido precisamente como su despliegue. La empresa resulta magna y era prevista en este punto de la investigación fenomenológica, pero, a la vez, advertida como extremadamente dificil "por mucho tiempo". Precisamente, el tiempo transcurrido y las múltiples exploraciones que han acumulado información sobre la esfera de lo inaparente hacen posible reconocerla, hoy, en los límites del territorio fenomenológico, con la tarea por delante de integrarla en una red de desarrollos que den cuenta de

\footnotetext{
${ }^{24}$ Sostiene Husserl: “...puesto que veo que la evidencia de la esfera fenomenológica no es menos que la de la matemática, $\mathrm{y}$, además, porque veo que todos los problemas filosóficos convergen sobre el suelo nutricio de la fenomenologia, me limito durante decenios a la fenomenología pura y a la formación de su método, a la solución de sus auténticos problemas fundamentales, en lugar de volverme predominantemente a los problemas filosófico-religiosos y a otros problemas de la trascendencia que están tanto más próximos a mi corazón. / ... tengo una gran inclinación hacia la mística alemana, cuya religiosidad interior me atrae mucho" (Carta del 5.IX.1917, en: Hua D III/3, pp. 418ss.).

${ }^{25}$ Hua I, p. 169.
} 
lo dado en todas sus dimensiones. Desde esta perspectiva, las visiones criticas y las denuncias de falta de radicalidad de la fenomenología husserliana constituyen los estudios esperados para el despliegue total del método que, desde esta perspectiva, se resignifican, pierden su carácter rupturista y resultan instancias positivas en la tarea de dar cuenta del arco completo de fenomenicidad.

\section{Los problemas del cuarto principio}

Retornemos al texto henriano. Tras esta revisión critica, el estudio analiza el tratamiento de la novedad marioniana de un cuarto principio dictaminando que está igualmente afectado de incertidumbre, pues la fórmula de una proporción entre reducción y donación lleva de nuevo al primer principio. La reducción tomada en su sentido fenomenológico es una reducción al aparecer ${ }^{26}$. Henry objeta que, más allá de la pretensión de radicalidad, la reducción al don mantiene una diferencia entre dos modos de manifestación y no da cuenta de un modo univoco de la fenomenalidad pura, única vía para explicitar su relación con el $\operatorname{ser}^{27}$. Así, esta versión de la fenomenología repetiría la confusión entre el aparecer del mundo y la esencia del aparecer que se remonta a los orígenes de la tradición, tal como está expuesto en el segundo principio ${ }^{28}$.

Henry dice sobre él que contiene una doble tesis contradictoria: por una parte, tiende a la universalidad de todo fenómeno; por otra, deja la fenomenalidad como factor de la intencionalidad y solo será fenómeno lo que pueda intencionarse. De este modo, solo es fenómeno el objeto trascendente: "es la trascendencia de este objeto, su puesta en distancia lo que constituye la fenomenalidad como tal"29. Un entorno como este no podrá captar el fenómeno de la vida, sino que quedará siempre al margen, pues el fenómeno se reduce a la donación intencional bajo el modelo de la visión, donde la intuición "no es más que un nombre para esta trascendencia" 30 y carga sobre si "esta eliminación inconsciente pero radical de la vida" 31 .

Por esta vía se reclama a la fenomenología husserliana una exigencia desmedida sobre el fenómeno, que implica, en suma, la negación de un ser

${ }^{26}$ Cf. Henry, M., Phénoménologie de la Vie, p. 84.

${ }^{27}$ Cf. ibid., p. 85.

${ }^{28}$ Cf. ibid.

29 "C'est la transcendance de cet objet, sa mise à distance que constitue la phénoménalité comme telle" (ibid, p. 86). Cf. Lavigne, L., Michel Henry: pensé de la vie et culture contemporaine, París: Beauchesne, 2014, pp. 67-80.

30 "n'est que'un nom de cette transcendance” (Henry, M., Phénoménologie de la Vie, p. 86)

31 "cette élimitation inconsciente mais radicale de la vie" (ibid.) 
que no sea intuible y, por tanto, una restricción indebida del campo disciplinar que anula la investigación del tipo de aparecer que sostiene todo el resto. Con otros términos, Henry reclamaria que no se haya demarcado claramente el terreno de un estrato de excedencia que apunte a una fenomenología de lo inaparente. Según hemos dicho, tal cosa no solo es posible, sino esperable en el plexo general del sistema fenomenológico general, de manera que hasta aquí no habría motivos para impugnaciones que lleven a puntos de ruptura.

Cuando Henry dice que "el segundo principio, en verdad el primero, es un crimen" ${ }^{32}$, subraya que el modo de dar cuenta de los fenómenos excluye el fenómeno de la vida y es, por tanto, su negación, es decir, un crimen, una falta de dimensiones catastróficas contra la Archi-Revelación del aparecer mismo que monta el edificio epistemológico sobre un asesinato ${ }^{33}$. No hay, sin embargo, riesgo de que la fenomenología se asiente en una morgue. Bastaría indicar que los dispositivos de la fenomenología estática y la fenomenología genética no son los adecuados para pensar los rasgos de un fenómeno cuya excedencia requiere de los métodos propios de la fenomenología de lo inaparente.

Como ejemplo de esta búsqueda, que consideramos dentro y no fuera de los límites de la fenomenología que llama "histórica", Henry despliega el camino para observar la cuestión de los principios. En la línea de una fenomenología material, acusa a Husserl de no plantear una materia fenomenológica impresional (une matière phénoménologique impressionnelle) que escape a la estructura intencional y evite los efectos de una reducción puramente negativa y ocultadora de "nuestra relación con el ser en tanto que nuestra propia vida" 34

El cuarto principio sugerido por Marion tendría, así, la ventaja de señalar los dos conceptos clave de la fenomenología en una relación de proporcionalidad que restituiría -o daría, en rigor, por vez primera- a la reducción una función positiva. De nuevo, deberíamos decir que esta fórmula tiene la ductilidad suficiente para funcionar en varios niveles, pues está orientada a dar cuenta de la potencia de la reducción. Henry afirma que la donación "lejos de limitar, de restringir o de omitir, de 'reducir', la reducción abre y da. ¿'Y qué da?”35. Esta

\footnotetext{
32 "le second príncipe, en vérité le premier, est un meurtre" (ibid.)

${ }^{33}$ Sobre la relación entre vida y violencia, $c f$. Thélot, J., "Vie et violence. Esquisse pour une généalogie du monde", en: Grégori, J. y otros (eds.), La vie et les vivants: (Re-)lire Michel Henry, Lovaina: PUL, 2013, pp. 425-440.

34 "notre relation à l'être en tant que notre propre vie" (Henry, M., Phénoménologie de la Vie, p. 88). Cf. Audi, P., Michel Henry: Une trajectoire philosophique, París: BBLL, 2006, esp. pp. 37 y 234

35 "loin de limiter, de restreindre ou d'omettre, de 'réduire' donc, la réduction ouvre et donne. Et que donne-t-elle?" (ibid.).
} 
formulación vale, evidentemente, para los contextos husserlianos que hemos visto. De hecho, está en la base de los procedimientos de doble reducción previstos en el caso de la vía psicológica. Alli precisamente se advierte con claridad la proporción creciente de donación y patencia fenomenológica entre el resultado de la reducción que ofrece los fenómenos de la psicología fenomenológica y el espectro que se abre con la reducción trascendental ${ }^{36}$.

Henry dice que Husserl no diferencia en La idea de la fenomenología el aparecer y lo que aparece, lo cual corrompe las nociones de donación absoluta y autodonación. Por ello, plantea que la fenomenología estuvo siempre presa, desde el surgimiento de la noción de reducción en 1905, de un equívoco óntico ${ }^{37}$. Así, critica el diseño de la reducción diciendo que no debe establecer "una división entre contenidos asegurados sobre los cuales se podría fundar por ejemplo el conocimiento de otros reputados dudosos"38, operando sobre un modelo de vía cartesiana que oculta el concierto de otras vías relacionadas con los modos regresivos. Nota bien que "no es jamás el contenido cierto, que es evidente, lo que es susceptible de proponerse como un fundamento para el conocimiento sino solamente lo que lo hace evidente y así, a saber, la evidencia misma" 39 . Esto no contraviene los principios de la fenomenología husserliana y expresa el sentido de ir a las cosas mismas. Es cierto que en fenómenos con excedencia no alcanza la matriz de la intencionalidad tal como funciona en los modelos estático, genético y generativo, pero eso no implica en modo alguno la salida del plexo general, sino solo, de nuevo, la indicación de la necesidad de contar con la fenomenología de lo inaparente como instancia que descubra la donación originaria (décuvre la donation originelle) ${ }^{40}$.

\footnotetext{
${ }^{36}$ Cf. Hua I, p. 16; Hua IX, p. 294; y Hua VI, p. 212 y la discusión sobre las vías en Kern, I., "The Three Ways to the Transcendental Phenomenological Reduction in the Philosophy of Edmund Husserl", en: Elliston, F. y otros (eds.), Husserl: Exposition and Appraisals, Notre Dame: University of Notre Dame Press, 1977, pp. 126-149; Luft, S., "Husserl's Theory of the Phenomenological Reduction: Between Life-World and Cartesianism", en: Research in Phenomenology, 34 (2004), pp. 198-234; y Staiti, A., "The Pedagogic Impulse of Husserl's Ways into Transcendental Phenomenology", en: Graduate Faculty Philosophy Journal, v. XXXIII, 1 (2012), pp. 39-46.

37 Cf. Henry, M., Phénoménologie de la Vie, p. 89. Sobre los elementos que morigeran esta afirmación, $c f$. González Di Pierro, E., "Gegebenheit y Donation: dos modos de dación fenomenológica. La crítica de Marion a Husserl. Coincidencias y divergencias”, en: Devenires, 12 (2011), pp. 153-165 e Inverso, H., Fenomenología de lo inaparente, Buenos Aires: Prometeo, 2018, pp. 31-47.

${ }^{38}$ Henry, M., Phénoménologie de la Vie, p. 89.

39 “Ce n'est jamais le contenu certain, ce qui est évident, qui est susceptible de seproposer comme un fondement pour la connaissance mais seulement ce qui le rend évident et ainsi à savoir l'évidence elle-même" (ibid., p. 90).

40 Ibid.
} 
A esta presentación general, Henry suma una serie consideraciones con carácter metodológico. Mientras todo lo visto reenvía a lo no visto, todo lo dado a lo no dado, de modo que toda donación se revela como provisoria, rodeada de horizontes y potencialidades irrealizadas, en la vida "todo está ahí entero a cada instante" ${ }^{41}$ porque esta donación última no tiene aspectos ni horizontes de cumplimiento y su materia fenomenológica es la afectividad, el pathos. No es el ver que pone en distancia lo que le es propio, sino un ver patético que se orienta a la reducción radical a la inmanencia pura, de la cual se dice que "no solo no olvida ni resta nada sino que es solo por ella que lo que pone entre paréntesis recibe sus propiedades particulares, mientras que el ver, la intuición, la evidencia abandonados a sí mismos no las explican"42.

Esta reducción radical a la inmanencia pura, que la fenomenología omitió, es la garantía de que no haya pérdida, mientras que la reducción a la evidencia no logra dar cuenta de la donación misma. En este contexto, Henry enfatiza la pertinencia de una reducción a la inmanencia y caracteriza como ilusión de la filosofia contemporánea el "creer que el ser definido fenomenológicamente como un Dimensional extático encuentre en ello una condición fenomenológica suficiente" 43 , es decir, que la trascendencia sea una esencia autónoma ${ }^{44}$, frente a lo cual cabe plantear que "el ser del aparecer, y especialmente el ser, no puede aparecer más que en tanto el aparecer aparece en sí mismo y en tanto que tal"45. Notemos que la "condición fenomenológica suficiente" es un factor que depende de la tematización efectuada, de modo tal que las bases latentes pueden recibir un análisis, pero esto no invalida los resultados de otros enfoques. En este sentido, no es preciso operar con una reducción a la donación en todos los casos, sino solo en aquellos en que la pregunta apunte a la dimensión última que funda todas las demás.

\footnotetext{
41 "tout est là tout entier à chaque instant" (ibid., p. 91). Cf., a propósito de la relación entre esta idea y el cuarto principio, Lagrut, B., "Deux réductions radicales? Le principe 'autant de réduction, autant de donation', chez Jean-Luc Marion et Michel Henry”, en: Grégori, J. y otros (eds.), La vie et les vivants: (Re-)lire Michel Henry, Lovaina: Presses Universitaires de Louvain, 2013, pp. 181-194.

42 "non seulement elle n'oublie ni ne retranche rien mais que c'est par elle seulement que ce qu'elle a mis entre parenthèses reçoit ses propriétés particulières, tandis que le voir, l'intuition, l'évidence laissés à eux-mêmes ne les expliquent nullement" (ibid., p. 92).

43 "croire que l'Être défini phénoménologiquement comme un Dimensional extatique trouve en celui-ci une condition phénoménologique suffisante" (ibid.)

44 Cf. ibid., p. 93.

45 Ibid. Cf. Mehl, E., "Auto-affection et cogito. Sur le cartesianisme de Michel henry", en: Ebbersmeyer, S. (ed.),
} 
Las conexiones que establece Marion con la filosofia primera y sus modelos históricos invita a comparar esta idea con los planos de análisis que ya distinguía Aristóteles ${ }^{46}$ : se puede hacer filosofia primera atendiendo a las causas, la ontología, la sustancia, pero también desde la teología, que apela a mecanismos ajenos al resto que dejan buena parte de sus dispositivos sin función, pues en ese plano no hay materia y por tanto no hay potencia. Sin embargo, esta redefinición local no invalida los demás los elementos, sino que los comprende como herramientas válidas para dar cuenta de los objetos en las otras perspectivas de la filosofia primera.

En síntesis, es impropio aplicar los parámetros de la teología a todos los planos, dado que eso debilita la capacidad misma del sistema, la cual reposa en su complementariedad de enfoques. Incluso, cabe sostener que, igual que en el terreno fenomenológico, cada uno de los análisis de los otros planos remite al plano fundante debido al marco teleológico que, con sentidos diversos, opera en ambos $\operatorname{casos}^{47}$. La referencia no es antojadiza, dado que Henry asocia el equivoco que apela al ser para pensar el aparecer con el modelo griego, que toma como parámetro del aparecer el aparecer del mundo. El cuarto principio estaría, entonces, puesto al servicio de mostrar la donación más originaria.

En este clima, Henry retoma dos cosas: por un lado, las críticas de Marion a Husserl, que quedaría preso del estudio de las objetualidades sin llegar a detectar el problema del ser; por otro, a Heidegger, que detectaría como problema el olvido del ser, pero avanzaría hundiéndose irremisiblemente en el ser en el mundo y perdiendo toda posibilidad de vislumbrar la donación originaria. Así, afirma: "la superación necesaria de la analítica existencial desplegada en Ser y Tiempo tiene por motivo principal la condición óntica cuyo pensamiento del ser sigue siendo constantemente prisionero"48.

Por esta vía, acompaña Henry los planteamientos marionianos que tematizan la "llamada" y la "reivindicación" que subordinan el ser a algo más esencial. Dice, por ello, que "la llamada del ser es simplemente su surgimiento

\footnotetext{
${ }^{46}$ Cf. por ejemplo, en Marion, J.-L., Acerca de la donación. Una perspectiva fenomenológica, Buenos Aires: UNSAM-Baudino, 2005, p. 39.

${ }^{47}$ Sobre la teleología insita en la fenomenologia, cf. Rizzacasa, A., "The Epistemology of the Sciences of nature in Relation to the Teleology of Research in the Thought of the Later Husserl", en: Tymieniecka, A.T., (ed.), Analecta Husserliana, vol. 9, Dordrecht/Boston/Londres: Reidel, 1979, pp. 73-84 y Miettinen, T., The Idea of Europe in Husserl's Phenomenology: A Study in Generativity and Historicity, Helsinki: Multiprint, 2013, pp. 233-382.

48 "le dépassement nécessaire de l'analytique existentiale déployée dans Sein und Zeit a pour principal motif le préalable ontique dont la pensée de l'être demeure constamment prisonnière" (ibid., p. 95).
} 
en nosotros, es el abrazo en el cual él se da a nosotros al mismo tiempo que nos hace ser. Por esto, no habría nada sin esta irrupción triunfal de una revelación que es aquella del Absoluto"49. Esta figura es inescindible de la del tedio, como posibilidad de no escuchar la llamada.

De este modo presenta la reducción radical como una puesta fuera de juego del ser y una escucha de la "forma pura de la llamada" 50 . En tanto forma pura del ser, como "fulguración de un aparecer que nos sumerge y que, en cuanto que fulgura, nos hace ser al mismo tiempo que él" ${ }^{51}$, pone en primer plano la esencia patética de la Vida.

\section{La versión marioniana de Siendo dado}

La cuestión de los principios es igualmente abordada por Marion en Siendo dado, donde, tras su sugerencia de un contra-método que limite la actividad del polo subjetivo para potenciar la mostración de lo que aparece, surge la pregunta sobre el modo en que concibe el principio de un método que pide perder la iniciativa. Para este tratamiento, se remonta al texto de Henry que acabamos de revisar. A pesar de variar el sistema de remisiones a los principios, mantiene su carácter y número, y coincide en la duda acerca de su coherencia y capacidad para asegurar el acceso a la manifestación. Así, se propone mostrar cómo la adición del cuarto principio, fruto de su propia producción, resuelve los problemas de ambigüedad que parecian presentarse.

Desde su perspectiva, la formulación "a tanto aparecer, tanto ser" pretende restablecer la dignidad óntica del aparecer dándole primacía, pero lo opone al ser, en una situación que termina reponiendo la metafísica ${ }^{52}$. La segunda formulación, "a las cosas mismas", somete el aparecer a las cosas, que serían lo que son de modo autónomo, marcando una primacía del ser sobre el aparecer. Si es así, el aparecer mostraría menos de lo que debe de las cosas

\footnotetext{
49 “L'appel de l'être, c'est tout simplement son surgissement en nous, c'est l'étreinte en laquelle il se donne à nous en même temps qu'il nous donne d'être. Ainsi n'y aurait-il rien sans cette irruption triomphale d'une révélation qui est celle de l'Absolu” (ibid., p. 96).

50 "forme pure de d'appel" (ibid., p. 99).

51 “a fulguration d'un apparaître qui nous submerge et qui, en tant qu'il fulgure, nous fait être en même temps que lui” (ibid., p. 100). Sobre la figura de fulguración del aparecer, véase Kühn, R., "Michel Henry's Radical Phenomenology", en: Archives de Philosophie, 74 (2011), pp. 65-93 y Borsch-Jacobsen, M., "L’inconscient malgré tout", en: Jean G. y J. Leclercq (eds.), Empreintes philosophiques, Lovaina: Presses Universitaires de Louvain, 2014, pp. 123-158.

$52 C f$. Fisette, D., "Phénoménologie et métaphysique: remarques à propos d'un débat récent", en: Narbonne, J-M. y L. Langlois (eds.), La Métaphysique, París: Vrin/ Presses de l’Universite Laval, 1999, pp. 91-116.
} 
que lo preceden ${ }^{53}$, minando el aparecer de defecto, lo cual permitiría conectar todo este planteamiento con la vinculación entre el aparecer y la apariencia entendida como opuesta a la realidad. Se trata, por el contrario, de invertir esta relación. Estas objeciones, que coinciden básicamente con las henrianas, llevan a rechazar estas dos fórmulas como elementos para caracterizar la fenomenología.

La tercera formulación, el principio de todos los principios, sostiene, según vimos, que "toda intuición donadora originaria es fuente de derecho para el conocimiento". De este modo, todo lo que se nos ofrece originariamente en la intuición, en su efectividad carnal, debe ser simplemente recibido como se da, pero sin rebasar los límites en los que se da. Este principio es reconocido como un dispositivo que libera de la exigencia metafísica de un fundamento, dado que basta con la intuición de un fenómeno, y libera también de una recaída en la analitica kantiana, porque no hacen falta a priori conceptuales ni formas puras de intuición ${ }^{54}$. El precio, señala Marion, es que la intuición se convierte en medida de la fenomenicidad ${ }^{55}$. Esa restricción nos remonta a las quejas de Heidegger a propósito de una limitación epistemológica que resulta insuficiente para dar cuenta de la facticidad.

Frente a esto Marion propone reavivar el vínculo entre reducción y donación que se remonta a Husserl, como surge del tratamiento a propósito de este punto en La idea de la fenomenología. Marion sostiene que el principio "a tanta reducción, tanta donación” permite aclarar los otros: la aparición no se da por el mero hecho de aparecer, sino en la medida en que se reduce a su donación a la conciencia. De este modo, "solo la reducción permite reconducir a la instancia que recibe la donación"56. Con esto se suprimiría la ambigüedad del segundo principio: volver a las cosas no implica ningún tipo de realismo precrítico, sino la reducción a lo trascendente de las vivencias tal como se dan a la conciencia, es decir, tal como en estas el fenómeno se da en persona. En segundo lugar, la reducción suspendería lo fraudulento, las "teorias absurdas", y excluiría la asunción de toda trascendencia real, con lo cual desaparece la ambivalencia

${ }^{5}$ Cf. Marion, J.-L., Étant donné. Essai d’une phénoménologie de la donation, p. 20.

54 Cf. ibid., p. 21.

${ }^{5} C f$. ibid., p. 21. Esto es así porque se convierte en un a priori, dado que sin intuición no hay donación, porque puede faltar, porque el principio no aclara los grados, porque la intuición restringe la fenomenicidad a una acepción limitada y está sometida al ideal de la representación objetivante, o porque este principio interviene antes y sin la efectuación de la reducción, pero sin reducción ningún procedimiento es fenomenológico.

56 Ibid., p. 26. 
del primer principio: parecer equivale a ser en tanto el aparecer se reduce a él mismo $^{57}$. En tercer lugar, si la intuición merece un privilegio, no lo debe al éxito del cumplimiento de la intención, sino a su carácter de intuición donadora.

En este punto, Marion identifica el límite que afectaría al principio de todos los principios y radicaría en que la intuición, en tanto donadora, funciona como "fuente de derecho" de la fenomenicidad en todos los casos en que los fenómenos dependen del ék-stasis y de la trascendencia. En donde no dependen, si los hay, la intuición no aportaría nada y la donación se ejercería sin intuición. En este sentido, la expresión "sin rebasar los límites en que se da" es ambigua, dado que apunta más a los límites de la intuición que a los de la donación, y resulta contradictoria porque, si la intuición soporta límites, la donación no los tiene, ya que lo que se da, en tanto que dado por donación reducida, se da por definición absolutamente. Así, el cuarto principio indicaría el sentido en que debe ser comprendido el principio de todos los principios, señalando que la donación se cumple por la reducción ${ }^{58}$.

Esta puntualización sirve a los efectos de diferenciar con claridad los distintos estratos, para mostrar que la relación entre intuición y donación no es la misma en cada una y que efectivamente hay una diferencia notable en el plano de la fenomenología de lo inaparente. El planteamiento íntegro, que constituye un aporte relevante para mostrar la relación y consistencia de los principios, indica de su funcionamiento en todos los planos comprendiendo las peculiaridades que adquieren en cada territorio fenomenológico. Así como "las cosas" de la fenomenología son distintas en cada uno, lo son también los modos del aparecer y las condiciones para ser fuente de derecho que los rige.

Podríamos agregar, a propósito del principio de todos los principios, que su valor reside no solo en lo que formula a propósito de la función de la reducción, sino en que aclara el resto señalando que, en rigor, están contenidos en él. En efecto, afirma que, en régimen de reducción, el resto de los principios ganan sentido y aplicabilidad. El genitivo de su fórmula resulta de este modo literal, por lo cual el principio de todos los principios, consideramos, debe ir en primer lugar, "antes" que los otros. Con este cambio de orden y el principio de

\footnotetext{
57 Cf. ibid., p. 27.

58 E. Tardivel desarrolla una serie de críticas a este principio, a propósito del modo en que concibe la reducción y su relación con el mundo y la donación, orientadas a acercar el dispositivo general a la hermenéutica ( $C f$. Tardivel, E., "Monde et donation. Une révision du quatrième principe de la phénoménologie", en: Revue de métaphysique et de morale, v. LXXXV, 1 (2015), pp. 121-160). Sin detenernos en la discusión de detalle, la adición de un modo de reducción no trascendental en este contexto oscurece el horizonte general.
} 
todos los principios encabezando la lista, se accede primero a la descripción de la correlación intencional, que lleva ínsita la invitación de la fórmula "a las cosas mismas" que sería, así, el segundo principio. Esta progresión se cierra con la fórmula "a tanto aparecer, tanto ser", acompañada de su explicitación en el cuarto principio, de modo tal que resulta un énfasis que no agrega nuevos datos.

4. La fórmula zu den Sachen selbst y las cosas de la fenomenología de lo inaparente

Del concierto de principios, el que Henry lista como tercero, la fórmula “a las cosas mismas", es, de lejos, el más mencionado como rasgo sintetizador epistemológico de la fenomenología. En sí mismo encierra, además, una concisión que ha levantado dudas sobre su capacidad para revelar su sentido a quienes no conozcan ya de antemano el planteamiento general. En efecto, Heidegger resumió bien en los Prolegómenos a una historia del concepto de tiempo la pregunta esperable: ¿a qué cosas mismas se refiere Husserl?59. La respuesta más directa pareciera ser "a los fenómenos", es decir, a aquello que se da como correlato de un acto intencional. Sin embargo, ya hemos visto que hay regiones donde la donación se da bajo regímenes diferentes, por lo cual comienzan a ensayarse respuestas variadas, que van desde la mención de la descripción de la experiencia hasta la objeción sobre los términos oscuros en los que está trazada la fórmula ${ }^{60}$.

G. Vargas Guillén insiste en la importancia de tener en cuenta el marco intelectual en el que, en la bisagra entre los siglos XIX y XX, varios grupos propugnaban vueltas hacia momentos o figuras del pasado como guía para la investigación. En ese contexto, la fórmula "a las cosas mismas" resulta contraria a todo escolasticismo y apelación a la autoridad y cifra la idea última de reducción de lo dado a la subjetividad constituyente del sentido ${ }^{61}$.

Esta caracterización es útil para nuestro recorrido porque el mismo principio, en un contexto donde se trata de revisar los alcances de la noción de sujeto, pasará a significar simplemente una reducción a lo dado, en todo caso desde la subjetividad, pero con el énfasis puesto en la manifestación autónoma

\footnotetext{
59 Heidegger, M., GA 20, p. 104.

${ }^{60}$ Un ejemplo de la primera opción es la caracterización de Steinbock, A., Home and Beyond: Generative Phenomenology After Husserl, Illinois: Northwestern University Press, 1995, p. 289; de la segunda, la posición de Zirión en la discusión que relevaremos.

61 Vargas Guillén, G., En torno a la fenomenología de la fenomenología: la pregunta por el método, Bogotá: UPN, 2012.
} 
del fenómeno. Una primera cuestión atiende a la relación entre la fórmula y la fenomenología como expresión de sus rasgos propios. Este aspecto del valor sintético de la fórmula ha dado lugar a un intercambio interesante entre A. Zirión Quijano y A. Xolocotzi con textos numerosos y argumentaciones de peso. No nos detendremos aquí en su reconstrucción, sino que tomaremos solamente uno de los textos de Zirión, en tanto discute la fórmula en juego respecto de los alcances de su aplicación, para considerar la variación de aspectos que puede presentar si se la enfoca desde las distintas dimensiones de la fenomenología, lo cual nos acercará nuevamente a la peculiaridad de "las cosas" de la fenomenología de lo inaparente.

La lectura de Zirión señala algunos rasgos que atañen a la pertinencia fenomenológica del lema "a las cosas mismas". En un primer trabajo de 1987, publicado dos años más tarde, revisa sus apariciones: las Conferencias de Paris ${ }^{62}$ y su pasaje paralelo en $\mathrm{CM}^{63}$; las menciones en Filosofia primera $\mathrm{I}^{64} \mathrm{y}$, mucho antes, en Ideas I, $\S 19^{65}$ (donde se menciona la expresión Sache selbst, pero no el lema, de lo cual infiere que, en la filosofia de Husserl, no siempre tiene valor de principio o de versión sucinta del principio de todos los principios) ${ }^{66}$. En el artículo "La filosofia como ciencia rigurosa" (1910), Husserl sostiene que "el incentivo para la investigación no tiene que provenir de las filosofias sino de las cosas y los problemas" ${ }^{67}$. En este sentido, el planteamiento inicial consistiria en un llamado epistemológico de carácter ético que invita al ejercicio autónomo de la razón y la liberación de prejuicios ${ }^{68}$.

En un trabajo posterior que profundiza esta lectura, se señala la variedad de fenomenologías y la dificultad de acercarse al método apelando al principio de "a las cosas mismas", el cual resultaría, más bien, un obstáculo69. Con esto, ataca directamente la tradicional idea de que en este principio estaría cifrado el rasgo común de las distintas variantes, como afirma Spiegelberg en la versión del artículo de la Enciclopedia Británica sobre "Fenomenología" que ocupa desde 1991 el lugar del controvertido texto que Husserl redactó, en una

62 Hua I, p. 6.

63 Hua I, p. 51 y p. 188.

${ }^{64}$ Hua VII, p. 22 у p. 32.

65 Hua III/I, p. 41.

66 Hua III/I, p. 51.

67 Hua XXV, p. 61.

68 Zirión Quijano, A., "La palabra de las cosas. Reflexiones sobre el lema 'A las cosas mismas", en: A. Zirión Quijano (comp.), Actualidad de Husserl, México D.F.: UNAM, 1987.

69 Zirión Quijano, A., "El llamado a las cosas mismas y la noción de fenomenología", en: Escritos de filosofia, 43 (2003). Las referencias que siguen corresponden todas a este texto. 
de sus versiones preliminares, en colaboración con Heidegger. Este principio no sería el que todo fenomenólogo suscribe, sin importar cuánto haya variado el resto del dispositivo de abordaje de las cosas. En rigor, expresaría solo una intención y no permitiría distinguir a la fenomenología de las otras ciencias.

El planteamiento sostiene que Husserl no la usa en ese sentido, que se remonta más bien al §7 de Ser y Tiempo, y responde, por tanto, a una apropiación heideggeriana. Además, el llamado, además, tiene una raíz ética de responsabilidad filosófica y la noción de cosa es general y formal y no se confunde con sentidos específicos que la noción de Sache tiene dentro del dispositivo íntegro. No implica por sí mismo, tampoco, ningún procedimiento asociado con la reducción fenomenológica o eidética ni de análisis intencional. Efectivamente, Heidegger conecta, en La filosofia y la tarea del pensar, dicho lema con el principio de todos los principios, movimiento que no sería necesario para Husserl ya que lo concibe como un principio de todo el conocimiento y no solo de la fenomenología. Estrictamente, habría que decir que, si la fenomenología va a ser el fundamento de todas las ciencias, los principios de la fenomenología se aplican al resto, y el hecho de que el ejemplo aducido sea el de un investigador de la naturaleza no va en contra de su encuadre fenomenológico.

Zirión cita a Husserl cuando critica al empirismo por entender a las cosas mismas como un fundamento del conocimiento en la experiencia y dice que, si el lema fuera propio de la fenomenología, Husserl estaría criticando al empirista por no practicar fenomenología. En rigor, Husserl objeta que no se reconozca que la ciencia debe tomar sus principios y fundamentos de la fenomenología y dejar de buscarlos en la experiencia. Se pide a las ciencias que no renieguen de la fenomenología y cuenten, al contrario, con su tarea: dado que habrá de darles fundamento, les brindará también sus principios.

Más allá de que localmente, en sus regiones, puedan tener sus propias legalidades, en el punto de la fundamentación las ciencias no pueden ser antifenomenológicas. Si se aduce que Meditaciones cartesianas presenta la fórmula como un primer principio metódico, cabe aclarar que lo es para la figura del "filósofo que comienza", es decir, como actitud general ineludible ${ }^{70}$. En la mención de Investigaciones lógicas II, Husserl se refiere a un retroceder a las cosas mismas pensando en conceptos o juicios, y no en el mundo precientífico, como lo interpreta, por ejemplo, Merleau-Ponty ${ }^{71}$. Cabe notar que esto refuerza la idea de que el lema no es inútil, sino un virtual catalizador de posi-

\footnotetext{
${ }^{70}$ Cf. Hua I, p. 54.

71 Cf. Hua XIX/1, p. 10.
} 
ciones fenomenológicas, dado que no solamente hay diferencias entre Husserl, Heidegger y Merleau-Ponty respecto de qué se entiende por cosas y cómo se va o retrocede hacia ellas, sino que, en otros planteamientos fenomenológicos posteriores, precisamente por la redefinición de aspectos ligados con el grado de radicalidad y las nociones de método, fenómeno y sujeto, el lema de ir a las cosas mismas varía su sentido pero no su validez. Su núcleo expresa la fenomenología en su sentido amplio de filosofia y de filosofia primera y, por tanto, con dominio sobre los sentidos últimos del conocimiento íntegro.

Más tarde, en el prefacio a la edición de 1913, Husserl marca el valor de los aportes fenomenológicos en su ir a las cosas mismas sin perderse en discusiones sobre puntos de vista ${ }^{72}$. El sentido de las cosas se aclara en el prólogo a la segunda edición de la sexta de las Investigaciones lógicas, donde afirma que la investigación se inclina hacia las cosas mismas y su presencia intuitiva en actitud fenomenológico-eidética sobre la conciencia pura y es, por ello, la única investigación que puede dar una teoría de la razón ${ }^{73}$. Zirión insiste en que hay intuiciones y responsabilidad fuera de la fenomenología. En este sentido, no todas son fenomenológicas, sino que tienen que ver con el conocimiento en general, del que se ocupa la fenomenología. Esto se reforzaría con la afirmación de que "la experiencia de una cosa es certeza original del ser, es la certeza del yo experimentante de estar junto a la cosa misma, de captarla a ella misma"74.

En esta lectura, si el ir a las cosas mismas fuera propio de la fenomenología, la descripción del ajuste entre pensamiento y cosa referiría a eventos solo fenomenológicos y no a cualquier proceso cognoscitivo. Esto oculta, sin embargo, que la reducción no altera contenidos, de manera que al operar en el plano fenomenológico todo lo mundano está disponible y allí se revelan precisamente sus legalidades. No sorprende que deba aclararse el sentido en que, en La filosofía como ciencia rigurosa, Husserl pasa de marcar la relación con la intuición directa a indicar que esta intuición es la captación fenomenológica de la esencia. Esto apunta a señalar que es a través de la fenomenología que la filosofia alcanza su status como ciencia, pero se trata de una segunda tesis que no se sigue de la relación entre filosofia e intuición directa y que requiere justificación adicional. Dentro del sistema, sin embargo, es algo que no puede

${ }^{72}$ Cf. Hua XVIII, p. 9.

${ }^{73}$ Cf. Hua XIX/2, p. 535.

${ }^{74}$ Hua XIX/2, p. 600 y Hua VI, p. 496. 
desligarse. Efectivamente, en otras partes ambos elementos aparecen en combinación como, por ejemplo, en Crisis §56 y Filosofía primera I75.

Esta combinación que materializaría el lema quitándole lo formal es asociado por Zirión con la lectura de Heidegger, Fink y Waldenfels, que por su cercanía y peso no son para tomar a la ligera, como tampoco lo son los acuerdos que llevaron a la declaración del editorial del Jahrbuch en 1913, donde se dice que lo que une a los editores es un retorno a las fuentes primarias de la intuición. Zirión lee este pasaje diciendo que se referian a la filosofia en general en un nivel previo y más amplio que la fenomenología, pero las conexiones usuales y repetidas que hacen de la fenomenología una filosofia sin más o su método vuelven dudosa la diferencia y, si se acepta incluso que esa fue la intención, parece una definición extrañamente limitada si es que realmente querían incluir otras lineas. Por otra parte, una actitud de este tipo tiene poco sentido en una revista orientada a promover la investigación fenomenológica. Puede ser que la mención no haya ayudado a aclarar la noción de fenomenología, pero no implica que no quisiera hacerlo.

Al ir a la formulación de Ser y tiempo, se enfatiza, en esta lectura, que Heidegger aplica la fórmula a todo conocimiento, pero hay que contar con que se trata de un modo de ir hacia una explicación. En la filosofia de Heidegger, este giro está asociado muy usualmente al procedimiento de desvelamiento que muestra que detrás de algo que parece obvio no hay tal obviedad, sino un núcleo de sentido. La objeción consiste en que Heidegger desformalizaría el lema y entendería cosas como cosas que son, es decir, que materializaría el planteamiento y definiría un objeto para la fenomenología, con lo cual perdería sentido como expresión del principio de todo conocimiento científico y como llamado vocacional. A propósito de esto, cabe decir que la intención de Heidegger se aleja de la preocupación teórico-científica, por lo cual es un elemento esperable y, en todo caso, resulta vocacional ya no en general, sino orientado a la filosofia.

En El final de la filosofía y la tarea del pensar, Heidegger vuelve a la conexión entre el lema y el principio de todos los principios y eso lleva a que la "cosa" de la filosofia sea la subjetividad absoluta. Fink, por su parte, se refiere a la fórmula diciendo que es la máxima fenomenológica de la investigación y que debe ser entendida formalmente como aplicable a todo lo que pueda ser traído a la vista ${ }^{76}$. Zirión discute esta idea diciendo que no todos los fenómenos

\footnotetext{
75 Cf. Hua VII, p. 70.

76 Fink, E., "Die phänomenologische Philosophie Edmund Husserls in der gegenwärtigen Kritik", en: Kant-Studien, v. XXXVIII, 1-2 (1933).
} 
son objetos posibles de fenomenología porque un estudio fenomenológico de los objetos de la geometría no sería el mismo que la geometría de los mismos objetos. Sin embargo, debe agregarse que, en tanto la fenomenología le da su fundamento a prioriy los objetos de la geometría están presentes bajo régimen de reducción, son objetos también fenomenológicos.

De hecho, Zirión sostiene que "a las cosas" implica el compromiso fenomenológico de análisis intencional. La pregunta aquí es si esto acaso implica una des-formalización. Podriamos responder que no. A diferencia del caso heideggeriano, donde hay redefinición de la cosa en relación con el ser, en el enfoque husserliano nunca se sale de la cosa como fenómeno y su amplitud es universal, aunque claramente pueda tematizarse de las maneras más variadas. Zirión alega que "si esto es algo que por supuesto no puede ser visto en la máxima a la primera mirada, entonces la máxima misma es absolutamente inútil para señalarlo o significarlo" 77 , pero nada obliga a que un principio deba ser transparente para quien se inicia en un campo y, muy por el contrario, la fenomenología requiere de conversiones radicales para que comience a tomar forma la totalidad, como surge de la figura del filósofo que comienza aludido en las Conferencias de Londres, en las Conferencias de París y en sus secuelas.

Así, el principio tiene plena validez si es una guía reguladora para quienes están instalados en la disciplina. Aun más, podría decirse que no importa si primero la máxima se malentiende y se crea el equívoco naturalizante contra el que tantas veces advierte Husserl, dado que si se siguen las derivas del filósofo que comienza eso se revelará prontamente como un camino imposible y la redefinición de las cosas llevará a la correlación intencional y a la conciencia pura. No hace falta pensar, entonces, que el principio se definió primero como formal y luego se lo desformaliza para aplicarlo a la fenomenología. "A la correlación intencional misma" no es una segunda máxima que instancia la primera en el terreno de la fenomenologia, sino una expresión alternativa del mismo principio.

Finalmente, Zirión retoma la interpretación de Waldenfels del lema en relación con lo que llama el impulso a la cosidad (Zug zur Sachlichkeit), que lo materializaría, porque "algo más que un concepto puramente formal de la cosidad (Sachlichkeit) se necesita para poder afirmar que la fenomenología tiene siempre que tematizar, junto con el contenido cósico (Sachgehalt), el modo de acceso (Zugangsweise) o que lo que ella busca es el lógos de los phenómena"78.

\footnotetext{
${ }^{77}$ Zirión Quijano, A., "El llamado a las cosas mismas y la noción de fenomenología", en: Escritos de filosofia, 43 (2003), pp. 157-182, p. 177.

${ }^{78}$ Ibid.
} 
Es una idea interesante, precisamente, para considerar que, desde distintos diseños, la fenomenología considera siempre el lugar a partir del cual la realidad llega a la visión y al lenguaje.

La lectura de Zirión advierte que no es conveniente absorber el lema en la fenomenología para no perder la guía y el correctivo más efectivo, lo cual sirve para mantener presente la relación de la fenomenología con el resto del conocimiento. Este recorrido resulta relevante porque sugiere que puede haber distintos modos de ir a las cosas mismas. La diferencia más clara se da entre la fenomenología, con su programa de ir a las cosas sin supuestos, y las ciencias positivas, que van hacia ellas sin deshacerse de sus prejuicios, lo cual implica una variación del modo en que las cosas mismas son concebidas. Es lo mismo que sucede, podríamos decir, entre las "cosas husserlianas" y las "cosas heideggerianas". Podría agregarse, entonces, que el recorte también varía si miramos la cosidad desde las distintas dimensiones de la fenomenología.

Esta variación estaría asociada directamente con el dispositivo de tematización de la fenomenología, que consiste, como hemos visto, en poner la atención sobre una cosa del mundo en el marco de un horizonte ${ }^{79}$. La tematización dicta el modo en que nos ubicamos en el plexo de cosas, en el mundo de la vida, y esa ubicación que tomamos respecto del objeto determina el modo en que algo se da ${ }^{80}$. Ante esta cuestión, A. Steinbock recurre a Derrida y sus consideraciones en “'Génesis y estructura' y la fenomenologia”, donde señala, precisamente, que estructura y génesis dan cuenta de aspectos distintos y resultan más adecuados cada uno en distintos $\operatorname{casos}^{81}$.

La tensión que se plantea es, entonces, si las cosas se muestran de acuerdo con la disposición con la que se las intenciona o, por el contario, si las cosas mismas en su apertura requieren un tipo específico de descripción. En este sentido, el planteamiento de Steinbock apunta a la prioridad del método o del fenómeno en la determinación de la clave de mostración del fenómeno. En un horizonte plenamente husserliano, si es preciso dar cuenta de sistemas de configuración, un enfoque estático resultará preferible, pero mostrará sus límites si el modo del fenómeno entraña devenir, origen o movimiento. Si se avanza hacia lo generativo, estos fenómenos advienen en un plexo de mayor

\footnotetext{
79 Cf. Hua VI, p. 327.

${ }^{80}$ Cf. Hua III/ 1, p. 362.

81 Steinbock, A., "Generativity and the Scope of Generative Phenomenology", en: Welton, D., The New Husserl: A Critical Reader, Indiana: Indiana University Press, 2003, p. 304 y Derrida, J., L'écriture et la différence, Paris: Seuil, 1967, cap. 5.
} 
complejidad atravesados por su emplazamiento en la historia, entre otros aspectos, y dejan abierto un campo de advenimiento de la excedencia ${ }^{82}$.

$\mathrm{El}$ punto es claramente relevante para nuestro estudio, dado que en la inapariencia los fenómenos presentan rasgos de initerabilidad que debilitan la posibilidad de una deliberación marcada sobre su abordaje, ya que adquiere peso su carácter de evento y la prioridad de su advenir. Del mismo modo, el polo subjetivo presenta una impronta de receptividad que va contra la determinación de una elección del modo en que se hará que el fenómeno se muestre. Si los ejercicios de cambio de perspectiva están a la mano en el caso de la estaticidad y la geneticidad, esto resulta más complicado en otros niveles.

En el caso del nacimiento analizado por Steinbock, para una mirada estática se trata de un fenómeno subliminal, dado que esta perspectiva no da cuenta del tiempo. Pasado y futuro son fenómenos límite, de modo que se puede hablar de retención y protención sin explicar cómo se forman. Desde la fenomenología genética, por su parte, el nacimiento no tiene suficiente donación fenoménica, pero sí aparece como tema para la fenomenología generativa, atendiendo precisamente al marco de intersubjetividad y de núcleos primarios familiares, así como a la importancia del nacimiento para la constitución del mundo ${ }^{83}$. Podemos agregar a eso que resulta igualmente tematizable por la inapariencia en su aspecto distintivo, esta vez atendiendo a su excedencia, dado que precisamente este rasgo que volvía a este fenómeno invisible en otros niveles es aquí el tematizado, lo cual redunda en una recuperación de fenómenos para la fenomenología.

\section{Corolarios}

Las líneas que podemos asociar con aportes a la fenomenología de lo inaparente suelen enfatizar la novedad de su campo, enfatizando precisamente que la discusión acerca de los principios y, entre ellos, el principio de "a las cosas mismas" se da en el plano de su extensión o no en los objetos de las ciencias, es decir, en un plano de fenomenicidad pobre o común (para usar terminología marioniana). Por el contrario, desde una perspectiva que incluya la inapariencia, "las cosas mismas" que se muestran se amplian en variedad y se fenomeniza,

\footnotetext{
${ }^{82}$ En rigor, no necesariamente hay que hacer intervenir la variable de progresión o superación que surge en algunos aspectos del trabajo de Steinbock, por ejemplo en Home and Beyond. Generative Phenomenology After Husserl, p. 43. En esta perspectiva importa la diferenciación de enfoques de los distintos dispositivos, al margen de su dimensión abarcativa.

${ }^{83}$ Cf. Steinbock, A., "Generativity and Generative Phenomenology", p. 308.
} 
de este modo, lo que quedaba latente. M. Henry señala la importancia de esta visión abarcadora diciendo: "la fenomenología desconfia de las explicaciones últimas, dedicándose en primer lugar a los problemas de la descripción. Pero una descripción que deja escapar lo esencial de la 'cosa misma', en este caso el pathos de toda intersubjetividad concreta, no puede legitimarse ni siquiera sobre el plano de la facticidad" 84 .

En este sentido, sea cual sea la posición sobre esta cuestión, la prioridad pasa por tener en cuenta que en sede fenomenológica, es decir, en lo que toca a la apropiación fenomenológica del lema "a las cosas mismas", esta fórmula apunta a dar cuenta de ellas de modo exhaustivo en el sentido de profundidad, pero también del carácter abarcativo, de modo que la fenomenicidad no quede cercenada. El dispositivo de principios avala el reconocimiento de diversos estratos fenomenológicos y la identificación de la inapariencia como plano que incluye desarrollos auto-definidos en el distanciamiento respecto de la matriz husserliana. Su integración potencia esos desarrollos tanto como los de la fenomenología en su vertiente fundante, abriendo campos fenoménicos relegado y muchas veces malentendidos.

Recibido: $18 / 01 / 2017$

Aceptado: 08/05/2018

\section{Bibliografia}

Audi, P., Michel Henry: Une trajectoire philosophique, París: BBLL, 2006.

Borsch-Jacobsen, M., "L'inconscient malgré tout", en: Jean, G. y J. Leclercq, Empreintes philosophiques, Lovaina: Presses Universitaires de Louvain, 2014.

Calcagno, A., "Michel Henry's Non Intentionality Thesis and Husserlian Phenomenology", en: Journal of the British Society for Phenomenology, v. XXXIX, 2 (2008), pp. 117-129.

Canullo, C., "Ekstasy of the World/Immanence of Life. Michel Henry reader of Husserl", en: Analecta Husserliana, Nueva York: Springer, 2003.

Fink, E., "Die phänomenologische Philosophie Edmund Husserls in der gegenwärtigen Kritik", en: Kant-Studien, v. XXXVIII, 1-2 (1933), pp. 319-383.

Fisette, D., "Phénoménologie et métaphysique: remarques à propos d'un débat récent", en: Narbonne, J-M. y L. Langlois (eds.), La Métaphysique, París: Vrin/Presses de 1’Universite Laval, 1999, pp. 91-116.

${ }^{84}$ Henry, M., Phénoménologie matérielle, p. 158. 
González Di Pierro, E., “Gegebenheit y Donation: dos modos de dación fenomenológica. La crítica de Marion a Husserl. Coincidencias y divergencias”, en: Devenires, v. XXII (2011), pp. 153-165.

Henry, M., L'Essence de la manifestation, París: PUF, 1963.

Henry, M., Philosophie et Phénoménologie du corps, París: PUF, 1965.

Henry, M., Phénoménologie matérielle, París: PUF, 1990.

Henry, M., Fenomenología material, Madrid: Encuentro, 2009.

Henry, M., Incarnation. Une philosophie de la chair, París: Éditions du Seuil, 2000.

Henry, M., Encarnación: una filosofía de la carne, Teira, J., Fernández, G. y R. Ranz (trads.), Madrid: Sígueme, 2001.

Henry, M., Fenomenología de la vida, Buenos Aires: UNGS/Prometeo, 2010.

Husserl, E., Gesammelte Werke-Husserliana, I-LXII., Biemel, W. y otros (eds.), Dordrecht: Springer (con anterioridad, Kluwer Academic Publishers y Martinus Nijhoff), 1950-2014.

Inverso, H., "La fenomenología de lo inaparente y el problema de las vías hacia el plano trascendental", en: Eidos, 26 (2016), pp. 43-73.

Inverso, H., "Más allá de la gnosis griega. El estoicismo y Michel Henry a propósito de la afectividad, la vida y la comunidad", en: Aufklärung: Revista de Filosofia, v. IV, 1 (2017), pp. 23-35.

Inverso, H., "Sobre la crítica de la presentación noemática de la alteridad en M. Henry y el despliegue de una fenomenología de lo inaparente”, en: Tópicos. Revista de Filosofia, v. LVII (2018), pp. 69-86.

Inverso, H., "Sobre un antiguo exilio de la luz. Los contactos obliterados entre la gnosis griega y la filosofia de M. Henry", en: Revista Alpha, v. XLVII (2018), pp. 121-133.

Inverso, H., "Polo subjetivo y toma de iniciativa. La fenomenología de lo inaparente y el contra-método marioniano", en: Revista Escritos, v. LVI (2018), pp. 63-82.

Inverso, H., Fenomenología de lo inaparente, Buenos Aires: Prometeo, 2018.

Kern, I., "The Three Ways to the Transcendental Phenomenological Reduction in the Philosophy of Edmund Husserl", en: Elliston, F. y otros (eds.), Husserl: Exposition and Appraisals, Notre Dame: University of Notre Dame Press, 1977, pp. 126-149.

Kühn, R., "Michel Henry's Radical Phenomenology", en: Archives de Philosophie, 74 (2011), pp. 331-346.

Lagrut, B., "Deux réductions radicales? Le principe 'autant de réduction, autant de donation', chez Jean-Luc Marion et Michel Henry”, en: Grégori, J. y otros (eds.), La vie et les vivants: (Re-)lire Michel Henry, Lovaina: Presses Universitaires de Louvain, 2013, pp. 181-194.

374 Lavigne, J., Michel Henry: pensé de la vie et culture contemporaine, París: Beauchesne, 2006.

Levinas, E., "Beyond Intentionality”, en: Montefiore, A. (ed.), Philosophy in France Today, Cambridge: Cambridge University Press, 1983, pp. 100-115.

Luft, S., "Husserl's Theory of the Phenomenological Reduction: Between Life-World and Cartesianism”, en: Research in Phenomenology, 34 (2004), pp. 198-234. 
MacDonald, P., Languages of Intentionality: A Dialogue Between Two Traditions on Consciousness, Londres: Continuum, 2012.

Marion, J.-L., Réduction et donation. Recherches sur Husserl, Heidegger et la phénoménologie, París: PUF, 1989.

Marion, J.-L., Étant donné. Essai d'une phénoménologie de la donation, París: PUF, 1997.

Marion, J.-L., Siendo dado. Ensayo para una fenomenología de la donación, Bassas, J. (trad.), Madrid: Sintesis, 2008.

Marion, J.-L., Figures de la phénoménologie, Paris: Vrin, 2012.

Mehl, E., "Auto-affection et cogito. Sur le cartesianisme de Michel Henry", en: Ebbersmeyer, S. (ed.), Emotional minds. The Passions and the Limits of Pure Inquiry in Early Modern Philosophy, Berlin: De Gruyter, 2012, pp. 31-49.

Miettinen, T., The Idea of Europe in Husserl's Phenomenology: A Study in Generativity and Historicity, Heksinki: Multriprint, 2013.

Popa, D., "Michel Henry, lecteur de Husserl. Apparence, phénoménalité et présence à soi", en: Cahiers philosophiques, v. III, 126 (2011), pp. 82-94.

Rizzacasa, A., "The Epistemology of the Sciences of nature in Relation to the Teleology of Research in the Thought of the Later Husserl”, en: Tymieniecka, A.T., (ed.), Analecta Husserliana, v. IX, Dordrecht/Boston/Londres: Reidel, 1979, pp. 73-84.

Staiti, A., "The Pedagogic Impulse of Husserl's Ways into Transcendental Phenomenology", en: Graduate Faculty Philosophy Journal, v. XXXIII, 1 (2012), pp. 39-46.

Stan, L., "Michel Henry: The Goodness of Living Affectivity", en: Stewart, J.B. (ed.), Kierkegaard and Exitentialism, Surrey: Ashgate, 2011, pp. 127-154.

Steinbock, A., Home and Beyond: Generative Phenomenology after Husserl, Illinois: Northwestern University Press, 1995.

Steinbock, A., "Generativity and Generative Phenomenology", en: Husserl Studies, v. XXII (1997), pp. 55-79.

Steinbock, A., "Husserl's Static and Genetic Phenomenology: Translator's Introduction to two Essays", en: Continental Philosophy Review, v. XXXI (1998), pp. 127-134.

Steinbock, A., "Generativity and the Scope of Generative Phenomenology", en: Welton, D. (ed.), The New Husserl: A Critical Reader, Indiana: Indiana University Press, 2003, pp. 289-325.

Tardivel, E., "Monde et donation. Une révision du quatrième principe de la phénoménologie”, en: Revue de métaphysique et de morale, v. LXXXV, 1 (2015), pp. 121-160.

Thélot, J., "Vie et violence. Esquisse pour une généalogie du monde", en: Grégory, J. y otros (eds.), La vie et les vivants: (Re-)lire Michel Henry, Lovaina: PUL, 2013, pp. 425-438.

Vargas Guillén, G., En torno a la fenomenología de la fenomenología: la pregunta por el método, Bogotá: UPN, 2012.

Walton, R., "Teleología y teología en Edmund Husserl”, en: Estudios de filosofía, 45 (2012), pp. 81-103. 
Zirion Quijano, A., "La palabra de las cosas. Reflexiones sobre el lema 'A las cosas mismas", en: Zirión Quijano, A. (comp.), Actualidad de Husserl, México D.F.: UNAM, 1989, pp. 99-123.

Zirión Quijano, A., "El llamado a las cosas mismas y la noción de fenomenología", en: Escritos de filosofia, 43 (2003), pp. 157-182. 